\title{
Calcitonin and vitamin $D_{3}$ have high therapeutic potential for improving diabetic mandibular growth
}

\author{
Mona A Abbassy ${ }^{1,2,3}$, Ippei Watari ${ }^{2}$, Ahmed S Bakry ${ }^{4,5,6}$, Takashi Ono ${ }^{2}$ and Ali H Hassan ${ }^{1}$
}

The goal of this study was to assess the effect of the intermittent combination of an antiresorptive agent (calcitonin) and an anabolic agent (vitamin $D_{3}$ ) on treating the detrimental effects of Type 1 diabetes mellitus (DM) on mandibular bone formation and growth. Forty 3-week-old male Wistar rats were divided into four groups: the control group (normal rats), the control C+D group (normal rats injected with calcitonin and vitamin $D_{3}$ ), the diabetic $C+D$ group (diabetic rats injected with calcitonin and vitamin $D_{3}$ ) and the diabetic group (uncontrolled diabetic rats). An experimental DM condition was induced in the male Wistar rats in the diabetic and diabetic $C+D$ groups using a single dose of $60 \mathrm{mg} \cdot \mathrm{kg}^{-1}$ body weight of streptozotocin. Calcitonin and vitamin $D_{3}$ were simultaneously injected in the rats of the control $C+D$ and diabetic $C+D$ groups. All rats were killed after 4 weeks, and the right mandibles were evaluated by micro-computed tomography and histomorphometric analysis. Diabetic rats showed a significant deterioration in bone quality and bone formation (diabetic group). By contrast, with the injection of calcitonin and vitamin $D_{3}$, both bone parameters and bone formation significantly improved (diabetic $C+D$ group) $(P<0.05)$. These findings suggest that these two hormones might potentially improve various bone properties.

International Journal of Oral Science (2016) 8, 39-44; doi:10.1038/ijos.2015.47; published online 18 December 2015

Keywords: type 1 diabetes mellitus; mandibular bone structure; mandibular bone formation; micro-computed tomography; bone; histomorphometry

\section{INTRODUCTION}

The prevalence of type 1 diabetes mellitus (DM) is considered a global health care crisis that is increasing in incidence. ${ }^{1}$ Intensive investigations have revealed that DM has detrimental effects on various body organs and tissues, and these effects may include alterations in bone and mineral metabolism, ${ }^{2-4}$ decreased bone density and increased fragility fractures, as well as poor bone healing and regeneration characteristics. ${ }^{5}$

Few studies have explored the effects of DM on craniofacial growth, although this field of study is of primary importance to health care professionals concerned with monitoring and treating craniofacial deficiencies, such as orthodontists and craniofacial surgeons. These studies showed that DM decreased mandibular bone formation, had a deleterious effect on osseous turnover, affected the histological integrity of the jaw bones due to alterations in the histomorphometric parameters, and affected the quality of bone structure, resulting in the stunting of its skeletal development. ${ }^{1-3}$ Moreover, previous studies have shown that diabetes affects the cephalometric measurements in individuals who are still growing. ${ }^{6}$ These results were confirmed when diabetes was introduced in growing animals and observed under strict experimental conditions, including the selection of the animals, feeding conditions and age. ${ }^{2,7}$ Another study showed that the detrimental effects of diabetes on craniofacial growth may be induced in the foetuses of diabetic mothers. ${ }^{6}$

Various lines of experimental treatment have been introduced ${ }^{8}$ to overcome the detrimental effects of DM on bone. Among these treatments, a theoretically attractive approach was suggested that includes the intermittent administration of an anabolic agent to promote bone formation and an antiresorptive agent that would prevent further bone loss. ${ }^{7,9}$ Evidence suggests that vitamin $\mathrm{D}_{3}$ can serve as the anabolic agent because it has a direct anabolic effect on bone and it increases the survival of osteoblasts. ${ }^{9-10}$ In contrast, calcitonin can be used as the antiresorptive agent through its action with its specific receptors, causing powerful inhibition of osteoclast activity; moreover, its role as a regulator of calcium homeostasis that involves bone resorption is well documented. ${ }^{9,11}$ Consequently, this may aid in improving the lines of treatment for the craniofacial growth deficiency observed in DM patients.

The hypothesis of the current study was that intermittent dosing of vitamin $\mathrm{D}_{3}$ and calcitonin can improve the detrimental effects of DM on the internal structure and the formation process of mandibular rat bone.

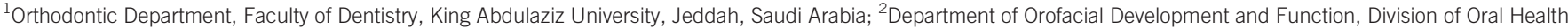

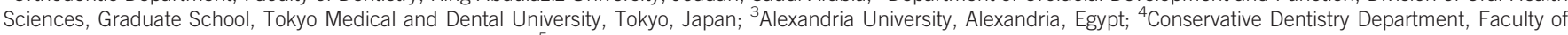

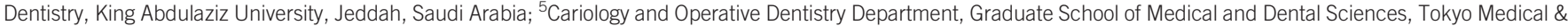
Dental University, Tokyo, Japan and ${ }^{6}$ Conservative Dentistry Department, Faculty of Dentistry, Alexandria University, Alexandria, Egypt 


\section{MATERIALS AND METHODS}

\section{Animals and experimental diabetic model}

Animal protocols were approved by the Institutional Animal Care and Use Committee of King Abdulaziz University, and the experiment was carried out under the control of the University's Guidelines for Animal Experimentation.

Forty 3-week-old male Wistar rats were used for this study. The rats were randomly divided into four groups: control group; the control group injected with calcitonin and vitamin $\mathrm{D}_{3}$ (control $\mathrm{C}+\mathrm{D}$ group); the diabetic group treated with calcitonin and vitamin $\mathrm{D}_{3}$ (diabetic $\mathrm{C}+\mathrm{D}$ group); and the diabetic group in which diabetes was introduced but no treatment was given (diabetic group). The rats in the control group and the control $\mathrm{C}+\mathrm{D}$ group were injected with single dose of $0.1 \mathrm{~mol} \cdot \mathrm{L}^{-1}$ sodium citrate buffer $(\mathrm{pH} 4.5)$, whereas the rats in the diabetic $\mathrm{C}+\mathrm{D}$ group and the diabetic group were injected intraperitoneally with a single dose of citrate buffer containing $60 \mathrm{mg} \cdot \mathrm{kg}^{-1}$ body weight of streptozotocin (STZ; Sigma, St Louis, MO, USA) ${ }^{12-13}$ to induce diabetes. Animals in all groups were fed a standard rodent diet (CE-2; Japan Clea, Shizuoka, Japan) with free access to water. Diabetes was determined in the diabetic $\mathrm{C}+\mathrm{D}$ group and the diabetic group by the presence of a high level of glucose in the urine and blood, and a positive urine test and a blood glucose level of $>200 \mathrm{mg} \cdot \mathrm{dL}^{-1}$ were considered as $\mathrm{DM}^{2,7}$ The rats in the control $\mathrm{C}+\mathrm{D}$ group and the diabetic $\mathrm{C}+\mathrm{D}$ group were subcutaneously injected with calcitonin (Sigma, St Louis, MO, USA), which was prepared in sterile saline with a concentration of $2.0 \mu \mathrm{g} \cdot \mathrm{mL}^{-1}$, and were dosed at with $1 \mathrm{~mL} \cdot \mathrm{kg}^{-1}$ body weight during the second and fourth weeks of the experiment. In addition, the rats in the control $\mathrm{C}+\mathrm{D}$ group and the diabetic $\mathrm{C}+\mathrm{D}$ group were injected subcutaneously with vitamin $\mathrm{D}_{3}, 1,25-(\mathrm{OH})_{2} \mathrm{D}_{3}$ (Cayman, Ann Arbor, MI, USA), which was prepared in ethanol $(96 \%)$ and diluted in $0.15 \mathrm{~mol} \cdot \mathrm{L}^{-1} \mathrm{NaCl}$, and were dosed with $0.025 \mu \mathrm{g} \cdot \mathrm{kg}^{-1}$ body weight ${ }^{14}$ during the first and third weeks of the experiment.

\section{Administration of calcein and preparation of sections}

All rats were injected subcutaneously with $50 \mathrm{mg} \cdot \mathrm{kg}^{-1}$ body weight of a calcein fluorescent marker on 21 and 28 days after STZ injection. At the end of the study, all animals were killed by transcardiac perfusion using $4 \%$ paraformaldehyde in $0.1 \mathrm{~mol} \cdot \mathrm{L}^{-1}$ phosphate buffer $(\mathrm{pH}$ 7.4). The right hemimandibles were dissected and fixed in the same solution. After being embedded in polystyrene resin (Rigolac; Nisshin EM, Tokyo, Japan), undemineralised ground frontal sections were processed to show the crown and both apices of the buccal and lingual roots of the lower second molar. We focused on the bone around the lower second molar because the second molar is centrally located within the mandibular arch, and the parallel alignment of the buccal and lingual roots made a precise reference when the frontal sections were produced. ${ }^{7,15}$

\section{Analysis of histomorphometric indices}

Histomorphometric bone indices of the periosteal surfaces of the alveolar and the jaw bones in all groups were evaluated using confocal laser scanning microscope (Carl Zeiss Jena, Jena, Germany) and a morphometry programme (LSM Image Browser; Carl Zeiss Jena, Jena, Germany).

The mineral apposition rate $\left(\mu \mathrm{m} \cdot \mathrm{d}^{-1}\right)$ and the bone formation rate $\left(\mu \mathrm{m}^{3} \cdot \mu \mathrm{m}^{-2} \cdot \mathrm{d}^{-1}\right)$, as previously described by Parrafit et al., ${ }^{16}$ were detected in the frontal sections of the lower second molar area.

The calcein-labelled surface (CLS; mm) was calculated as the sum of the length of double labels $(\mathrm{dL})$ plus one-half of the length of single labels (sL) along the entire endosteal or periosteal bone surfaces; i.e.,
CLS $=\mathrm{dL}+0.5 \mathrm{sL}$. The mineral apposition rate $\left(\mathrm{MAR} ; \mu \mathrm{m} \cdot \mathrm{d}^{-1}\right)$ was determined by dividing the mean of the width of the dLs by the interlabel time (i.e., 7 days). The bone formation rate $\left(B F R ; \mu \mathrm{m}^{3} \cdot \mu \mathrm{m}^{-2} \cdot \mathrm{d}^{-1}\right.$ ) was calculated by multiplying the MAR by the CLS. ${ }^{7}$ Based on the reference line along the long axis of the buccal root, the area superior to the root apex was considered to be alveolar bone, whereas the area inferior to the root apex was considered to be jaw bone. The lingual side of the mandibular bone was excluded in all specimens because of the existence of the incisor root, which may influence bone formation. The periosteal surfaces of the mandible were divided into four regions for analysis (Figure 1): region 1, alveolar crest (upper half of the tooth root, near the tooth crown); region 2, alveolar bone (lower half of the tooth root, near the root apex); region 3, buccal surface of the jaw bone; and region 4, inferior border of the jaw bone. For the measurement of the mineral apposition rate, the average of three inter-label widths at a $50-\mu \mathrm{m}$ interval were calculated for each sample. ${ }^{15}$

\section{Micro-computed tomography of the mandible}

All left mandibles were imaged using micro-computed tomography (CT; inspeXio SMX-90CT; Shimadzu Science, Tokyo, Japan) after removing only the soft tissue. The mandibular plane was set orthogonal to the sample stage, and imaging was performed. ${ }^{17}$ In summary, threedimensional (3D) images of each hemimandible were acquired with a resolution voxel size of $15 \mu \mathrm{m} \cdot \mathrm{pixel}^{-1}$. Raw data were obtained by rotating the sample stage $360^{\circ}$. Slice images were then prepared using multitomographic image reconstruction software (MultiBP; Imagescript, Tokyo, Japan). The resulting grey-scale images were segmented using a low-pass filter to remove noise and a fixed threshold to extract the mineralised bone phase. The volume of interest was drawn using a slicebased method starting from the first slice containing the crown of the first molar and moving dorsally 100 slices $^{18}$ in the area of the alveolar crest (between the buccal and lingual roots of the second molar at the cervical region) and the buccal surface of the jaw bone. ${ }^{15}$ Trabecular bone was carefully contoured on the first and last slices, whereas the intermediate slices were first interpolated by morphing. For observation and analysis of reconstructed 3D images, we used 3D trabecular structure analysis software (TRI/3D-BON; RATOC System Engineering, Tokyo, Japan). ${ }^{17}$ Reconstructed 3D images were prepared from slice

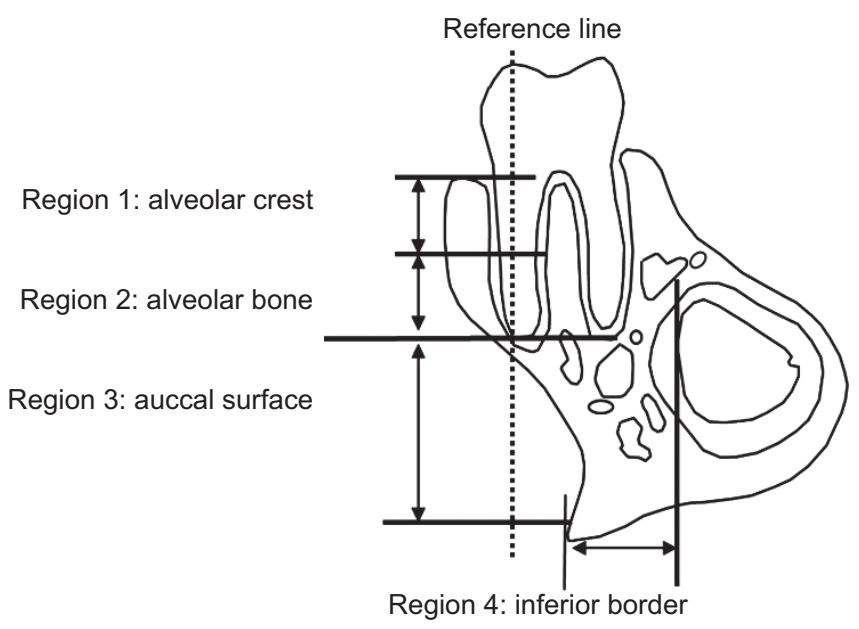

Figure 1 Schematic of the observation regions for dynamic bone histomorphometry. The periosteal surfaces were limited to four areas: alveolar crest (region 1: upper half of the tooth root, near the tooth crown), alveolar bone (region 2: lower half of the tooth root, near the root apex), buccal surface of the jaw bone (region 3 ) and inferior border of the jaw bone (region 4). 
images using the volume rendering method to analyse the microstructure of the bone. The following parameters were measured: tissue volume (TV), bone volume (BV), bone surface (BS), bone surface/bone volume $(\mathrm{BS} / \mathrm{BV})$ and bone volume fraction $(\mathrm{BV} / \mathrm{TV})$. Four properties of the trabeculae were evaluated: trabecular thickness (Tb.Th), trabecular number (Tb.N), trabecular separation (Tb.Sp) and trabecular space (Tb.S). ${ }^{17,19}$

\section{Histological analysis}

The left mandibles for all groups were decalcified in 10\% elhylene diaminetetraacetic acid (EDTA) solution ( $\mathrm{pH} 7.4$ ) for 5 weeks at $4{ }^{\circ} \mathrm{C} .{ }^{20}$ The specimens were then dehydrated in an ascending ethanol series and embedded in paraffin. Serial horizontal sections $(5 \mu \mathrm{m}$ thick parallel to the occlusal plane) were prepared using a microtome (Leica RM 2155; Leica, Nussloch, Germany). Five sections were used for histochemical staining of tartrate-resistant acid phosphatase (TRAP) activity. ${ }^{7}$ The sections were incubated for $30-60 \mathrm{~min}$ at $37{ }^{\circ} \mathrm{C}$ in a mixture of $0.8 \%$ naphthol AS-BI phosphate (Sigma, St Louis, MO, USA), 0.7\% fast red violet salt (Sigma, St Louis, MO, USA) and $50 \mathrm{mmol} \cdot \mathrm{L}^{-1}$ sodium tartrate diluted in $0.2 \mathrm{~mol} \cdot \mathrm{L}^{-1}$ sodium acetate buffer $(\mathrm{pH} 5.4) .^{7}$ The sections were examined under a light microscope. For the histomorphometric assessment of resorption, the number of TRAP-positive multinucleated cells (osteoclasts) on the distal surface of the alveolar bone adjacent to the mesio-buccal root of the second molar were counted in each $540 \mu \mathrm{m} \times$ $120 \mu \mathrm{m}$ area in five consecutive sections, at the middle third of the root selected at least $25 \mu \mathrm{m}$ from each specimen $(n=10)$ of each group. ${ }^{7,20}$

\section{Statistical analysis}

One-way analysis of variance and Tukey's post hoc test were used to compare the means of the MAR, the BFR, the bone parameters observed by micro-CT, and the number of TRAP-positive cells recorded in all groups. All statistical analyses were performed at a 5\% significance level using statistic software (v. 10; SPSS, Chicago, IL, USA).

\section{RESULTS}

\section{Analysis of histomorphometric indices}

Green fluorescent lines labelled with a calcein fluorescent marker at two different time-points showed that growth took place between days 21 and 28 in all groups (Figure $2 \mathrm{a}-2 \mathrm{~d}$ )

The rats in the diabetic group showed significant decreases in the MAR (Figure 2e) and BFR (Figure 2f) in regions 2, 3 and 4 compared with all other groups $(P<0.05)$. However, in the alveolar crest (region 1$)$, the MAR and BFR results did not show any significant difference among all groups $(P<0.05)$. The MAR and BFR rates in regions 2,3 and 4 in the diabetic $\mathrm{C}+\mathrm{D}$ group (diabetic rats injected with calcitonin and vitamin D) showed a significant increase when compared with those of the dia-
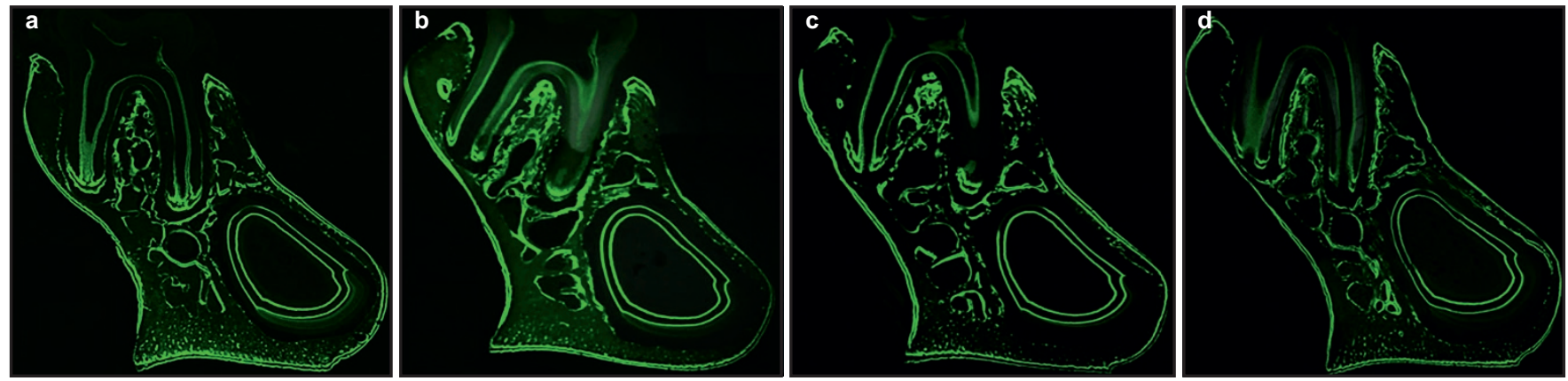

e

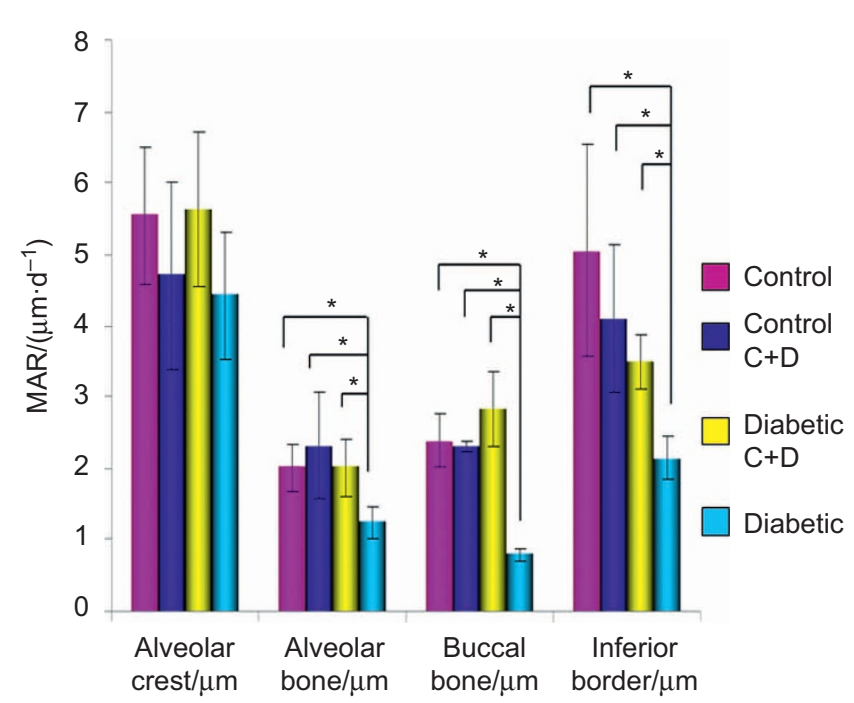

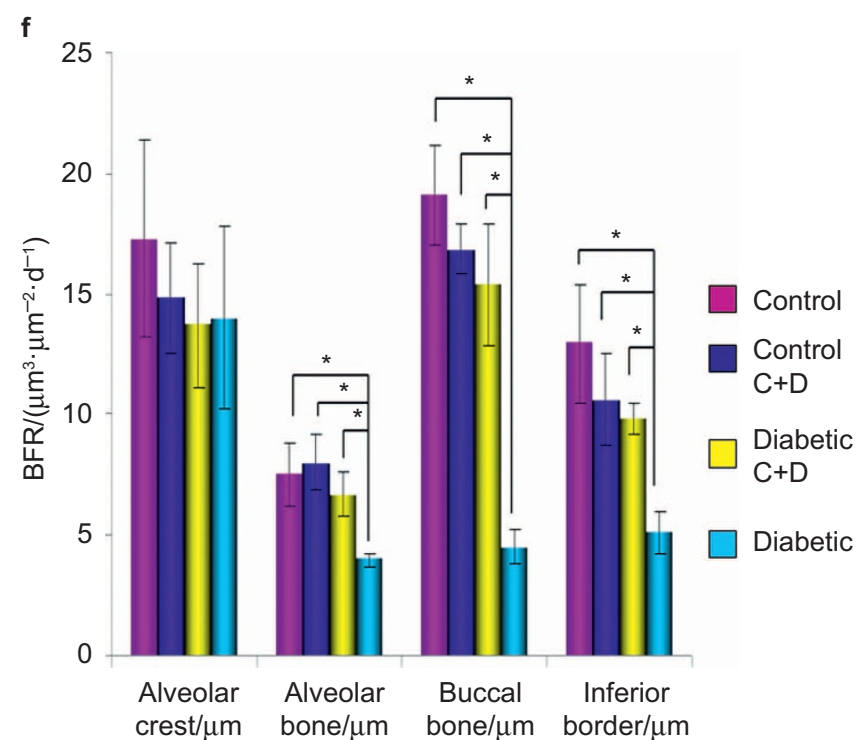

Figure 2 Frontal sections of the mandibular second molar area. (a) The control group; (b) the control C+D group; (c) the diabetic C+D group; (d) the diabetic group. Fluorescent labelling on the periosteal surface indicates new bone formation. (e) The changes in the MAR in regions 1-4 of the mandible among all groups. The data are expressed as the mean \pm SD. $n=10$ for each group. *Significant difference $(P<0.05)$. (f) The changes in the BFR in regions $1-4$ of the mandible among all groups. The data are expressed as the mean \pm SD. $n=10$ for each group. *Significant difference $(P<0.05)$. BFR, bone formation rate; MAR, mineral apposition rate; SD, standard deviation. 
Table 1 Three-dimensional bone microstructure analysis of alveolar bone imaged by micro-CT and evaluated using an automated image analyser

\begin{tabular}{|c|c|c|c|c|}
\hline Parameters & Control & Control C+D & Diabetic C+D & Diabetic \\
\hline (Bone surface/bone volume) $/ \mathrm{mm}^{-1}$ & $63.9 \pm 15.38^{a}$ & $68.8 \pm 4.54^{\mathrm{a}}$ & $53.9 \pm 7.98^{a}$ & $31.8 \pm 9.34^{b}$ \\
\hline (Bone volume/tissue volume)/\% & $46.2 \pm 1.02^{\mathrm{a}}$ & $50.7 \pm 2.58^{\mathrm{a}}$ & $59.4 \pm 3.1^{\mathrm{a}}$ & $22.1 \pm 11.56^{\mathrm{b}}$ \\
\hline Trabecular thickness $/ \mu \mathrm{m}$ & $34.3 \pm 4.50^{\mathrm{a}}$ & $29.67 \pm 1.86^{\mathrm{a}}$ & $36.11 \pm 6.14^{\mathrm{a}}$ & $22.2 \pm 1.78^{\mathrm{b}}$ \\
\hline Trabecular number/mm ${ }^{-1}$ & $14.4 \pm 2.56^{\mathrm{a}}$ & $17.4 \pm 1.3^{\mathrm{a}}$ & $16.3 \pm 2.89^{a}$ & $10.80 \pm 1.17^{b}$ \\
\hline Trabecular separation/ $\mu \mathrm{m}$ & $25.9 \pm 2.36^{a}$ & $28.4 \pm 2.98^{a}$ & $23.8 \pm 6.07^{\mathrm{a}}$ & $40.8 \pm 5.02^{b}$ \\
\hline Trabecular space/ $\mu \mathrm{m}$ & $71.18 \pm 11.5^{a}$ & $57.5 \pm 4.01^{a}$ & $62.5 \pm 10.44^{a}$ & $87.6 \pm 4.25^{b}$ \\
\hline
\end{tabular}

micro-CT, micro-computed tomography.

$P<0.05$, Same letters are statistically significant.

betic group $(P<0.05)$. Moreover, there was no significant difference in the MAR and BFR between the control group and the control $\mathrm{C}+\mathrm{D}$ group (normal rats injected with calcitonin and vitamin $\mathrm{D} ; P<0.05$ ).

\section{Micro-CT of the mandible}

All trabecular parameters in both the alveolar bone and the buccal surface of the jaw bone showed significant changes (Tables 1 and 2). Compared with all groups, the alveolar bone and the buccal surface of the jaw bone in the diabetic group showed significant deteriorations in $\mathrm{BV} / \mathrm{TV}$ and BS/BV. Moreover, the Tb.Th and Tb.N significantly decreased in both the alveolar and the buccal surfaces of the jaw bone in the diabetic group when compared with all other groups $(P<0.05)$.
Correspondingly, significantly higher Tb.Sp and Tb.S were revealed in both the alveolar and the buccal surfaces of the jaw bone for the diabetic group $(P<0.05)$. However, the values recorded for the BV/TV, BS/BV, Tb.Th, Tb.N, Tb.Sp and Tb.S of the alveolar bone and the buccal surface of the jaw bone in the diabetic $\mathrm{C}+\mathrm{D}$ group were not significantly different from the control group and the control $\mathrm{C}+\mathrm{D}$ group but were significantly different from the results in the diabetic group $(P<0.05)$.

\section{Histological analysis}

Bone resorption activity was assessed by counting the number of TRAP-positive cells on the distal surface of the alveolar bone adjacent to the mesio-buccal root of the second molar (Figure 3a-3e). The
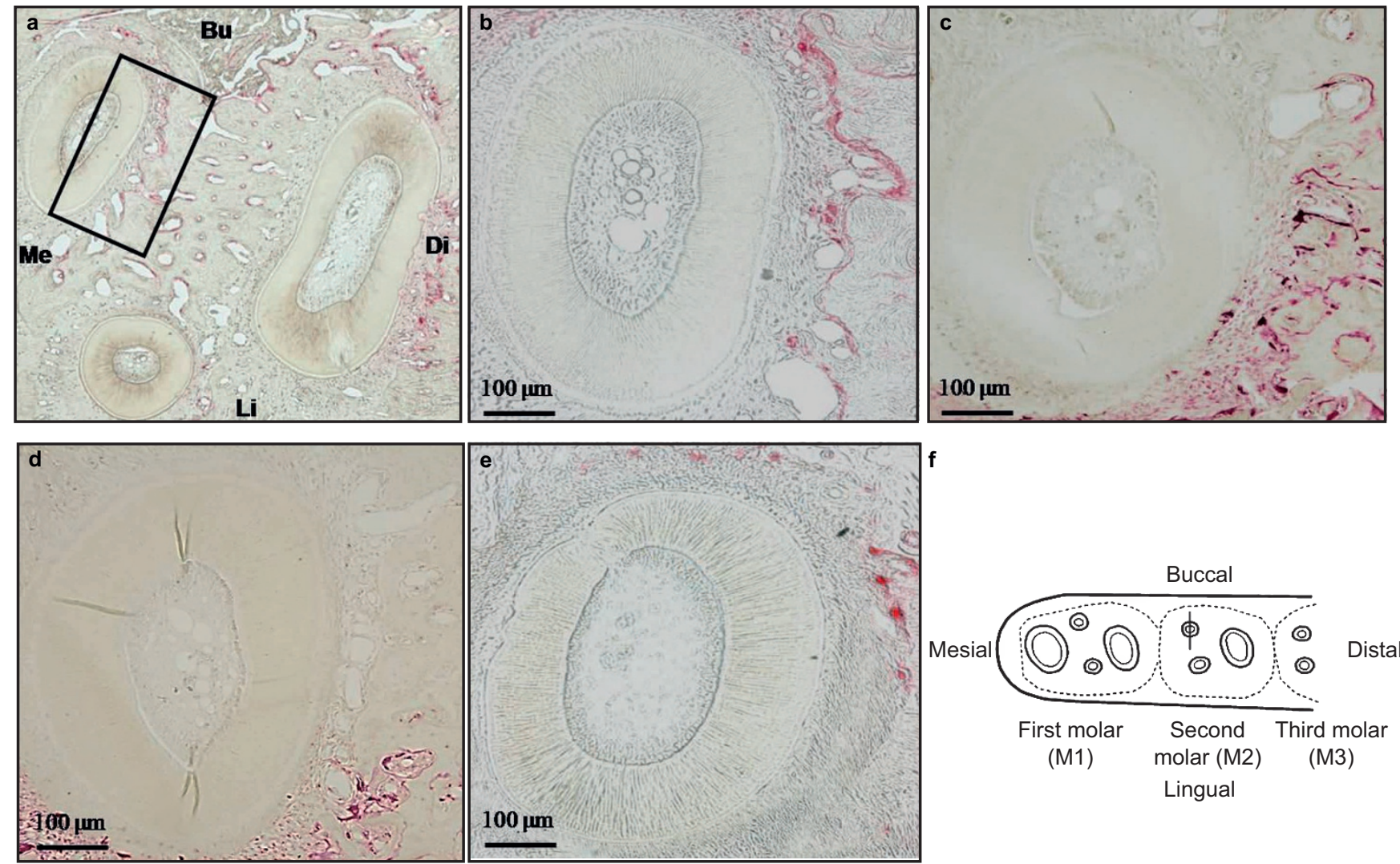

Figure 3 Osteoclast counts in a horizontal section of the mandibular second molar region stained with TRAP. (a) Low magnification photograph of the three roots of

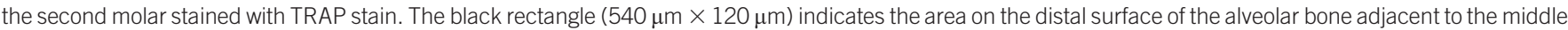

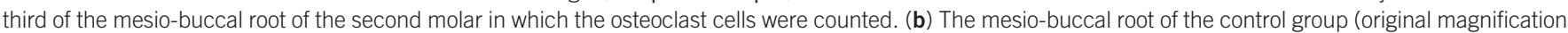

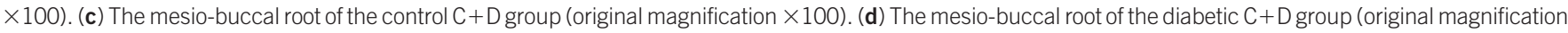

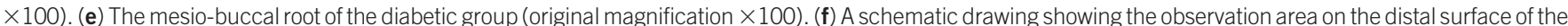
alveolar bone adjacent to the mesio-buccal root of the second molar in which the osteoclast cells were counted. Bu, buccal; Di, distal; Li, lingual; Me, mesial. 
Table 2 Three-dimensional bone microstructure analysis of the buccal surface of jaw bone imaged by micro-CT and evaluated using an automated image analyser

\begin{tabular}{|c|c|c|c|c|}
\hline Parameters & Control & Control C+D & Diabetic $C+D$ & Diabetic \\
\hline (Bone surface/bone volume)/mm ${ }^{-1}$ & $53.23 \pm 16.11^{a}$ & $40.39 \pm 5.17^{a}$ & $40.54 \pm 14^{a}$ & $86.64 \pm 4.67^{b}$ \\
\hline (Bone volume/tissue volume)/\% & $60.62 \pm 13.43^{a}$ & $72.2 \pm 2.75^{a}$ & $76.5 \pm 16.76^{a}$ & $37.3 \pm 4.15^{b}$ \\
\hline Trabecular thickness/ $\mu \mathrm{m}$ & $27 \pm 2.00^{\mathrm{a}}$ & $30.1 \pm 0.53^{a}$ & $30.28 \pm 1.15^{\mathrm{a}}$ & $21.5 \pm 2.82^{b}$ \\
\hline Trabecular number $/ \mathrm{mm}^{-1}$ & $18.45 \pm 0.17^{a}$ & $17.09 \pm 1.22^{\mathrm{a}}$ & $16.90 \pm 1.04^{\mathrm{a}}$ & $14.42 \pm 1.9^{b}$ \\
\hline Trabecular separation/ $\mu \mathrm{m}$ & $25.9 \pm 2.43^{\mathrm{a}}$ & $20.2 \pm 3.03^{\mathrm{a}}$ & $19.9 \pm 5.42^{a}$ & $40.9 \pm 4.93^{b}$ \\
\hline Trabecular space $(\mu \mathrm{m})$ & $54.40 \pm 0.29^{a}$ & $59.55 \pm 2.89^{a}$ & $59.89 \pm 3.25^{\mathrm{a}}$ & $68.9 \pm 7.06^{b}$ \\
\hline
\end{tabular}

micro-CT, micro-computed tomography.

$P<0.05$, Same letters are statistically significant.

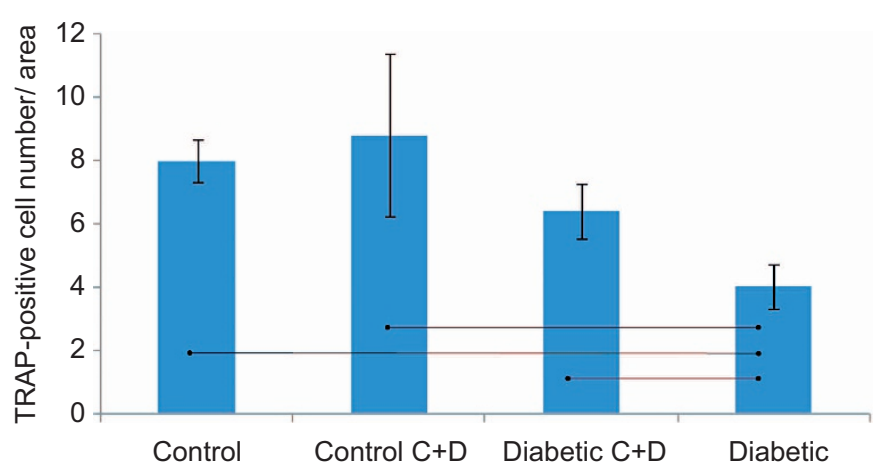

Figure 4 The number of TRAP-positive cells on the distal surface of the mesiobuccal root of the mandibular second molar. Values are mean $\pm S D$. Connected bars show the statistically significant differences among the four groups $(P<0.05)$, bars $=100 \mu \mathrm{m}$. SD, standard deviation.

diabetic group showed a significant decrease in the number of osteoclast cells (Figure 4) when compared with the control and the control $\mathrm{C}+\mathrm{D}$ groups. However, when the diabetic rats were treated with calcitonin and vitamin $\mathrm{D}_{3}$ in the diabetic $\mathrm{C}+\mathrm{D}$ group, the number of the osteoclast cells was not significantly different from the number of cells observed in either the control group or the control $\mathrm{C}+\mathrm{D}$ group $(P<0.05)$.

\section{DISCUSSION}

In this experiment, the intermittent dosing of vitamin $\mathrm{D}_{3}$ and calcitonin was adopted as a means to restore the detrimental effects of DM because these two hormones act on different cellular targets and could potentially have an additive beneficial effect. ${ }^{9}$ Previous research work has shown that the administration of vitamin $\mathrm{D}_{3}$ alone for prolonged periods or in high doses is not recommended due to the disturbances expected in calcemic activities; moreover, the application of calcitonin alone was found to be unable to restore detrimental effects on bone structure or mass caused by some systemic hormonal disturbances. $^{9,21}$

Rats between 3 and 8 weeks of age were used because this time period corresponds to the early growth stage in humans. ${ }^{2,22-23}$ To elaborate the mandibular bone growth changes during the short observation period adopted in the current study, the mandibular molar region was chosen. The mandibular molar exhibits remarkable eruption and has continuous root elongation along with growth, which has been reported to induce significant bone changes in these regions in growing rats. ${ }^{15} \mathrm{~A}$ calcein fluorochrome bone marker was injected twice to determine the MAR (an indication of the osteoblastic activities at the cellular level) and the BFR (which indicates any changes in the osteoblast number and, thus, bone formation at the tissue level). ${ }^{15}$
The significantly lower values for MAR and BFR observed in the diabetic group agree with previous studies that recorded diminished lamellar bone formation in the femurs of DM rats and may suggest an association between DM and the decreased number and function of osteoblasts. $^{24-25}$ Moreover, DM significantly decreased the BV/TV, Tb.Th and Tb.N and significantly increased the Tb.Sp and Tb.S in the diabetic group. This agrees with other studies that have suggested that glycaemic levels play an important role in modulating the trabecular architecture, especially in mandibular bone. ${ }^{5}$

The histometric evaluation of diabetic rats in the diabetic group confirmed the significant decrease in the number of osteoclast cells, which agrees with previous studies on the mandible $e^{7,26}$ and long bones $^{27-28}$ of DM rats.

All of the aforementioned findings suggest that diabetes lead to a reduction in the rate of bone turnover. However, calcitonin and vitamin $\mathrm{D}_{3}$ improved most of the deteriorated bone parameters observed in the diabetic $\mathrm{C}+\mathrm{D}$ group. Moreover, the osteoclasts cells significantly increased in the diabetic $\mathrm{C}+\mathrm{D}$ group compared with the osteoclast cells in the diabetic group. These improvements observed in the diabetic $\mathrm{C}+\mathrm{D}$ group may be attributed to the anabolic effect of vita$\min \mathrm{D}_{3}$ and to the antiresorptive effect of calcitonin.

The antiresorptive effect of calcitonin was previously suggested ${ }^{9,10,21,29}$ and was considered safe and capable of stabilizing or increasing bone mineral density. In addition, calcitonin is a powerful inhibitor of osteoclast activity with only a transitory action because it is quickly eliminated from the skeleton, circulation and extravascular fluids, allowing normal remodelling to take place. Finally, calcitonin could also have mild anabolic properties as a result of cross-reactivity among bone cell receptors for calcitonin, calcitonin gene-related peptide and amylin. ${ }^{30}$

The major anabolic effects of vitamin $\mathrm{D}_{3}$, which was administered to the diabetic $\mathrm{C}+\mathrm{D}$ group, include stimulation of calcium absorption (intestine), enhancement of calcium reabsorption (kidney), inhibition of parathyroid hormone synthesis and secretion (parathyroid glands), and regulation of bone resorption and bone formation (skeleton). ${ }^{29}$ Evidence suggests that vitamin $\mathrm{D}_{3}$ improves osteoblast survival and that this is the main mechanism underlying the anabolic bone effects of vitamin $\mathrm{D}_{3}$ regardless of calcium supplementation in the diet. ${ }^{9}$

Moreover, vitamin $\mathrm{D}_{3}$ was previously reported ${ }^{31}$ to increase the number of TRAP-positive cells in mice femurs; however, we hypothesise that the increase in TRAP-positive cells observed in the current experiment was not due to the direct effect of injecting the rats with vitamin $\mathrm{D}_{3}$ but rather to a general increase in the bone turnover in the diabetic $\mathrm{C}+\mathrm{D}$ group. Our hypothesis is based on the following. First, the positive improvement of most of the bone parameters observed in the diabetic $\mathrm{C}+\mathrm{D}$ group suggests that there was a concomitant increase in the osteoblasts along with the increase of the osteoclast cells. Second, a previous study reported that a rat model treated with 
calcitonin and vitamin $\mathrm{D}_{3}$ showed an increase in the osteoblast cell counts, so in the current experiment we hypothesised that there was a similar increase in the osteoblast cells, which may have expressed the nuclear factor kappa-B ligand (RANKL) that was capable of activating its osteoclasts cell receptor (RANK). ${ }^{32}$ The stimulation of RANK induces several key regulatory transcription factors and enzymes that are essential in the promotion, differentiation, proliferation, multinucleation, activation and survival of osteoclasts, ${ }^{32}$ which may explain the increased number of osteoclasts in the current experiment.

Furthermore, the application of calcitonin in this experiment limited the bone resorption activity exerted by the increased number of osteoclasts. This effect may be attributed to the binding of the calcitonin to specific receptors on the osteoclasts, leading to retraction of the osteoclasts from the bone surface and reduced production of acid and other proteolytic enzymes essential for the bone resorption process. $^{33}$ The effect of calcitonin ensured the diminishing of the catabolic effect exerted by the osteoclasts when compared with the anabolic effect exerted by the osteoblasts.

In conclusion, this paper suggested an important line of treatment for the stunted mandibular bone growth problems that clinicians concerned with observing and treating craniofacial complex defects may encounter; these problems were previously clinically observed by cephalometric analysis during orthodontic treatment of patients. ${ }^{6}$ Supplementation by antioxidants, which was previously suggested, ${ }^{8}$ and the hormonal treatment suggested in the current study may aid in decreasing the treatment period needed to enhance mandibular growth for orthodontic young patients during the mandible growth period. In the current study, diabetes caused various deteriorations in the mandibular bone parameters, as observed by micro-CT, histomorphometric and histological examinations; however, the intermittent injection of vitamin $\mathrm{D}_{3}$ and calcitonin in diabetic rats restored most of the examined bone parameters to their normal levels. The adopted hypotheses in this study were accepted.

\section{ACKNOWLEDGEMENTS}

This project was funded by the National Plan for Science, Technology and Innovation (MAARIFAH) - King Abdulaziz City for Science and Technology- the Kingdom of Saudi Arabia - award number (12-MED2735-03). The authors also, acknowledge with thanks Science and Technology Unit, King Abdulaziz University for technical support.

1 Giglio MJ, Lama MA. Effect of experimental diabetes on mandible growth in rats. Eur J Oral Sci 2001; 109(3): 193-197.

2 Abbassy MA, Watari I, Soma K. Effect of experimental diabetes on craniofacial growth in rats. Arch Oral Biol 2008; 53(9): 819-825.

3 Verna C, Melsen B. Tissue reaction to orthodontic tooth movement in different bone turnover conditions. Orthod Craniofac Res 2003; 6(3): 155-163.

4 Abbassy MA, Watari I, Bakry AS et al. Diabetes detrimental effects on enamel and dentine formation. J Dent 2015; 43(5): 589-596.

5 Thrailkill KM, Liu L, Wahl EC et al. Bone formation is impaired in a model of type 1 diabetes. Diabetes 2005; 54(10): 2875-2881.

6 El-Bialy T, Aboul-Azm SF, El-Sakhawy M. Study of craniofacial morphology and skeletal maturation in juvenile diabetics (Type I). Am J Orthod Dentofacial Orthop 2000; 118(2): 189-195.

7 Abbassy MA, Watari I, Soma K. The effect of diabetes mellitus on rat mandibular bone formation and microarchitecture. Eur J Oral Sci 2010; 118(4): 364-369.

8 Al Ghafli $\mathrm{MH}$, Padmanabhan R, Kataya $\mathrm{HH}$ et al. Effects of alpha-lipoic acid supplementation on maternal diabetes-induced growth retardation and congenital anomalies in rat fetuses. Mol Cell Biochem 2004; 261(1/2): 123-135.
9 Andresen $\mathrm{CJ}$, Moalli M, Turner $\mathrm{CH}$ et al. Bone parameters are improved with intermittent dosing of vitamin $D_{3}$ and calcitonin. Calcif Tissue Int 2008; 83(6): 393-403.

10 Erben RG, Bromm S, Stangassinger M. Therapeutic efficacy of $1 \alpha, 25$-dihydroxyvitamin $\mathrm{D}_{3}$ and calcium in osteopenic ovariectomized rats: evidence for a direct anabolic effect of 1 1 ,25-dihydroxyvitamin $D_{3}$ on bone. Endocrinology 1998; 139(10): 4319-4328.

11 Martin TJ. Calcitonin, an update. Bone 1999; 24(5 Suppl): 63S-65S.

12 Tein MS, Breen SA, Loveday BE et al. Bone mineral density and composition in rat pregnancy: effects of streptozotocin-induced diabetes mellitus and insulin replacement. Exp Physiol 1998; 83(2): 165-174.

13 McCracken MS, Aponte-Wesson R, Chavali R et al. Bone associated with implants in diabetic and insulin-treated rats. Clin Oral Implants Res 2006; 17(5): 495-500.

14 Gunness-Hey M, Hock JM, Gera I et al. Human parathyroid hormone (1-34) and salmon calcitonin do not reverse impaired mineralization produced by high doses of 1,25 dihydroxyvitamin $\mathrm{D}_{3}$. Calcif Tissue Int 1986; 38(4): 234-238.

15 Shimomoto Y, Chung CJ, Iwasaki-Hayashi Y et al. Effects of occlusal stimuli on alveolar/jaw bone formation. J Dent Res 2007; 86(1): 47-51.

16 Parfitt AM. Bone histomorphometry: standardization of nomenclature, symbols and units (summary of proposed system). Bone 1988; 9(1): 67-69.

17 Takada H, Abe S, Tamatsu Y et al. Three-dimensional bone microstructures of the mandibular angle using micro-CT and finite element analysis: relationship between partially impacted mandibular third molars and angle fractures. Dent Traumatol 2006; 22(1): 18-24.

18 Laib A, Rüegsegger P. Calibration of trabecular bone structure measurements of in vivo three-dimensional peripheral quantitative computed tomography with 28microm-resolution microcomputed tomography. Bone 1999; 24(1): 35-39.

19 Nakano H, Maki K, Shibasaki Y et al. Three-dimensional changes in the condyle during development of an asymmetrical mandible in a rat: a microcomputed tomography study. Am J Orthod Dentofacial Orthop 2004; 126(4): 410-420.

20 Yokoyama M, Atsumi T, Tsuchiya M et al. Dynamic changes in bone metabolism in the rat temporomandibular joint after molar extraction using bone scintigraphy. Eur J Oral Sci 2009; 117(4): 374-379.

21 Rizzoli R, Boonen S, Brandi ML et al. The role of calcium and vitamin D in the management of osteoporosis. Bone 2008; 42(2): 246-249.

22 Losken A, Mooney MP, Siegel MI. Comparative cephalometric study of nasal cavity growth patterns in seven animal models. Cleft Palate Craniofac J 1994; 31(1): 17-23.

23 Siegel MI, Mooney MP. Appropriate animal models for craniofacial biology. Cleft Palate J 1990; 27(1): 18-25.

24 Follak N, Klöting I, Wolf E et al. Histomorphometric evaluation of the influence of the diabetic metabolic state on bone defect healing depending on the defect size in spontaneously diabetic BB/OK rats. Bone 2004; 35(1): 144-152.

25 Shyng YC, Devlin H, Sloan P. The effect of streptozotocin-induced experimental diabetes mellitus on calvarial defect healing and bone turnover in the rat. Int J Oral Maxillofac Surg 2001; 30(1): 70-74.

26 Mishima N, Sahara N, Shirakawa M et al. Effect of streptozotocin-induced diabetes mellitus on alveolar bone deposition in the rat. Arch Oral Biol 2002; 47(12): 843849.

27 Glajchen N, Epstein S, Ismail F et al. Bone mineral metabolism in experimental diabetes mellitus: osteocalcin as a measure of bone remodeling. Endocrinology 1988; 123(1): 290-295.

28 Shires R, Teitelbaum SL, Bergfeld MA et al. The effect of streptozotocin-induced chronic diabetes mellitus on bone and mineral homeostasis in the rat. J Lab Clin Med 1981; 97(2): 231-240.

29 Holick MF. Resurrection of vitamin D deficiency and rickets. J Clin Invest 2006; 116(8): 2062-2072.

30 Wallach S, Rousseau G, Martin L et al. Effects of calcitonin on animal and in vitro models of skeletal metabolism. Bone 1999; 25(5): 509-516.

31 Tinkler SM, Williams DM, Johnson NW. Osteoclast formation in response to intraperitoneal injection of 1 alpha-hydroxycholecalciferol in mice. J Anat 1981; 133(Pt 1): 91-97.

32 Rachner TD, Khosla S, Hofbauer LC. Osteoporosis: now and the future. Lancet 2011 377(9773): 1276-1287.

33 Eriksson SA, Lindgren JU. Combined treatment with calcitonin and 1,25-dihydroxyvitamin $D_{3}$ for osteoporosis in women. Calcif Tissue Int 1993; 53(1): 26-28.

\section{(c) (i) $(-)$ This work is licensed under a Creative Commons Attribution-} cc) party material in this article are included in the article's Creative Commons license, unless indicated otherwise in the credit line; if the material is not included under the Creative Commons license, users will need to obtain permission from the license holder to reproduce the material. To view a copy of this license, visit http://creativecommons.org/licenses/ by-nc-nd/4.0/ 